\title{
Solvation Dynamics in Aqueous Reverse Micelles: A Computer Simulation Study
}

\author{
James Faeder ${ }^{\dagger}$ and Branka M. Ladanyi* \\ Department of Chemistry, Colorado State University, Fort Collins, Colorado 80523 \\ Received: February 16, 2001; In Final Form: August 13, 2001
}

\begin{abstract}
We present molecular dynamics simulation results for solvation dynamics of a simple diatomic solute in model reverse micelles of varying size. These results are compared to solvation dynamics of the probe in spherical cavities of the same size containing only water. Our simulations focus on the short-time dynamics of solvation, from 0 to $2 \mathrm{ps}$, a significant portion of which has not yet been accessed experimentally. On this time scale, the solvation response in reverse micelles becomes faster as the micelle size parameter, $w_{0}$, increases, in agreement with experiment, but most of the effect occurs in the slower, diffusive portion of the response. The short-time inertial dynamics, which account for over $70 \%$ of the response in all of the systems studied, appear to be quite robust even when the mobility of individual water molecules is greatly reduced. Decomposition of the nonequilibrium response functions demonstrates that the short time relaxation is dominated by water and occurs at the solute site where hydrogen bonds are broken. Analysis of the equilibrium solvation time correlation functions demonstrates that the linear response approximation is accurate for reverse micelles, but less so for the smooth cavities. Decomposing the equilibrium response into pair and singlemolecule contributions, we find that the pair contributions are larger in the reverse micelles and increase as $w_{0}$ decreases. This collective response appears to be much faster than the single molecule response and largely offsets the sharp reduction in single molecule mobilities. Another reason for the robustness of the inertial response may be the preferential location of our model probe outside the water layers closest to the interface. The relative magnitudes of fast and slow contributions to the solvent response for a particular chromophore may thus be sensitive to its location relative to the interface.
\end{abstract}

\section{Introduction}

Water in natural systems is often found in conditions very different from those of the bulk liquid. It is therefore of fundamental importance to characterize how the properties of water are affected by heterogeneity. Aqueous reverse micelles (RMs), which are nanoscopic pools of polar solvent in bulk solution stabilized by a surrounding layer of surfactant molecules, ${ }^{1-6}$ provide a simple model system in which to study how geometry and interfacial complexity affect the properties of water. They also have numerous practical applications-e.g., heterogeneous chemical and biochemical catalysis, drug delivery, and nanocluster synthesis. ${ }^{7-10}$

In a recent molecular dynamics (MD) computer simulation study, we have examined the structure and dynamics of water in the RM interior as a function of micelle size. ${ }^{11}$ The study focused on model RMs that were designed to represent those formed by the aerosol OT (AOT) surfactant. AOT is an anionic surfactant, with $\mathrm{Na}^{+}$as the counterion, that forms approximately spherical RMs, whose radius in the presence of a given oil phase depends solely on the ratio ${ }^{12,9}$

$$
w_{0}=\left[\mathrm{H}_{2} \mathrm{O}\right] /[\text { surfactant }]
$$

In agreement with many experimental measurements, ${ }^{9}$ water in the model RMs formed well-defined layers in the vicinity of the surfactant sulfonate headgroups, where water mobility was greatly reduced. Translational and rotational mobilities within a given layer also exhibited strong variations with micelle size.

$\dagger$ Present address: Theoretical Biology and Biophysics Group, MS K710, Los Alamos National Laboratory, Los Alamos, NM 87545.
These variations were related to both the increase in the size of the core region, where water properties are closer to the bulk, and to the increase with $w_{0}$ of the surface area per headgroup, ${ }^{12}$ which leads to higher mobility of the interfacial water. ${ }^{11}$

Studies of solvation dynamics (SD), the collective response of the solvent to a change in the charge distribution of a probe molecule, provide an additional way to characterize properties of water that are directly related to chemical reactivity. ${ }^{13}$ The solvation response of water, usually reported as the normalized response function $S(t)$, may in general be separated into two main components: a very fast initial decay that occurs on a sub-200 fs time scale due to inertial solvent motion, and a slower relaxation on a time scale of several hundred femtoseconds to picoseconds ascribed to diffusive solvent motion. Experiments $^{14,15}$ and MD simulations ${ }^{16-19,14,20-24}$ show that over $70 \%$ of the decay of $S(t)$ in bulk water occurs on a sub-200 fs time scale. In systems with interfacial water, the short time dynamics also appear to dominate the response, with the diffusive component accounting for about $10-30 \%$ of the total, but the diffusive portion decays over much longer times extending as far as the nanosecond regime. ${ }^{25}$ The origin and significance of this long time response are the subjects of ongoing debate.

Reverse micelles are an excellent system in which to investigate the effects of confinement and strong interfacial interactions on SD because they are well-characterized and their properties can be systematically varied. A number of studies have investigated SD in RMs through the time-dependent electronic spectra of chromophores dissolved in the interior region. ${ }^{26-39} \mathrm{SD}$ experiments have not yet accessed the shorttime ( $<200 \mathrm{fs}$ ) portion of the solvation response, $S(t)$, but the remaining response has been found to be markedly slower than

\subsection{1/jp010632n CCC: \$20.00 @ xxxx American Chemical Society} Published on Web 00/00/0000 PAGE EST: 10.8 
in bulk water and strongly dependent on RM size. ${ }^{26,36,31,33}$ On the other hand, the total amplitude of the solvation response, ${ }^{40}$ estimated by zero-time extrapolation of the fluorescence spectrum, ${ }^{41}$ decreases by only about $30 \%$ going from bulk water to the smallest reverse micelle, and the vast majority of the decay in $S(t)$ occurs during the early time period not accessed at the present time resolution. In addition, the magnitude of the early time response, the portion not captured in the experiments, appears to increase as $w_{0}$ becomes smaller, ${ }^{40}$ which seems to contradict the generally held view that solvation dynamics should slow down as solvent mobility is restricted. Simulation of the early time dynamics of solvation in RMs could help to resolve this apparent contradiction.

We report here a computer simulation study of SD in model RMs of varying size. The model is the same as the one we used in our earlier study of the water structure and mobility ${ }^{11}$ with the addition of a large diatomic probe molecule. To separate the effects on SD of water-surfactant interactions from the effects of confinement alone, we have also carried out MD simulations of SD in water confined within spherical hydrophobic cavities (HCs). A major goal of the present study is to shed light on the molecular aspects of the solvation mechanism in confined and heterogeneous systems. We accomplish this through the analysis of $S(t)$ in terms of contributions from different solvent components, different sites of the model chromophore, and the location of the chromophore with respect to the interface. The collective aspects of SD are studied by analyzing the contributions from intermolecular pair correlations, ${ }^{42,43}$ including interspecies cross-correlations, to the solvation time correlation function, the linear response approximation to $S(t)$.

Numerous simulations of SD in bulk liquid water have been carried out previously. ${ }^{16,17,19,14,44,20-24}$ To our knowledge, however, this is the first MD simulation of SD in confined water. Related to our work, Senepati and Chandra ${ }^{45}$ have recently examined SD of simple dipolar liquids in cavities, and even more recently Balasubramanian and Bagchi have reported results for SD of water near the surface of regular micelles. ${ }^{46}$

The remainder of the article is organized as follows: In Section II, we describe the model systems and give the simulation details. In Section III, we present our results for solvation dynamics in RMs and hydrophobic cavities of varying size. In addition to the MD results for $S(t)$, obtained from nonequilibrium MD simulation, we present the results of analysis of MD trajectory data to obtain mechanistic information, which we then use to account for the observed trends in the $w_{0}$ dependence of $S(t)$. Structural information and solvation time correlations obtained from equilibrium MD are included in this analysis. Sec. IV contains a summary of our main findings, a discussion of their implications for the effects of confinement on reaction dynamics, and possible model improvements.

\section{Interaction Model and Computer Simulation Details}

II.A. Interaction Model. We have modeled the interior of an aqueous reverse micelle as a rigid spherical cavity and treated only the surfactant headgroups, counterions, water, and the chromophore at a molecular level. Details of the model and the procedures used to produce equilibrated reverse micelles have already been presented elsewhere. ${ }^{11}$ The major difference between our model and an earlier model developed by Linse and Halle $\mathrm{H}^{47,48}$ is that in our model the headgroups protrude from the cavity boundary and are tethered only in the radial direction. These features allow the counterions to occupy bridging positions between the headgroups and permit the spacing between headgroups to be nonuniform. The radial positions of the headgroups are restricted using a harmonic potential

$$
u(d)=\frac{1}{2} k_{\mathrm{e}}\left(d-d_{\mathrm{e}}\right)^{2}
$$

where $d$ is the distance from the cavity wall, $d_{\mathrm{e}}=2.5 \AA$ is the equilibrium distance, and $k_{\mathrm{e}}=600 \mathrm{kcal} \mathrm{mol}^{-1} \AA^{-2}$ is the force constant. The interaction potential between the cavity and the free molecules on the interior is determined by assuming the region outside the cavity is composed of a uniform continuum of nonpolar molecules that interact with interior molecules through a Lennard-Jones (LJ) 6-12 potential. The resulting potential $^{11}$ is the spherical analogue of the standard 3-9 potential that is commonly used for flat interfaces. ${ }^{49}$ The molecular interactions inside the cavity are described in terms of potentials of the $\mathrm{LJ}+$ Coulomb form, with the interaction between sites of type $\alpha$ and $\beta$ on different molecules or ions given by

$u_{\alpha} \beta(r)=4\left(\epsilon_{\alpha} \epsilon_{\beta}\right)^{1 / 2}\left[\left(\frac{\sigma_{\alpha}+\sigma_{\beta}}{2 r}\right)^{12}-\left(\frac{\sigma_{\alpha}+\sigma_{\beta}}{2 r}\right)^{6}\right]+\frac{q_{\alpha} q_{\beta}}{4 \pi \epsilon_{0} r}$

where $\epsilon_{\alpha}$ and $\sigma_{\alpha}$ are the LJ well depth and diameter and $q_{\alpha}$ the partial charge for site $\alpha$. As indicated in the above equation, we are using the Lorentz-Berthelot combining rules to obtain the LJ parameters for unlike sites. The headgroups, which we denote as $\mathrm{Z}^{-}$, are represented as a single interaction site carrying a charge of $-e$, although work on removing this approximation by using a 4-site model for $\mathrm{SO}_{3}{ }^{-}$is currently under way. We use the SPC/E model for water. ${ }^{50} \mathrm{Na}^{+}$parameters were taken from a previous simulation of sodium dodecyl sulfate in water. ${ }^{51}$

The solute used in most of the recent SD experiments in AOT-based RMs ${ }^{29,31-33}$ has been coumarin 343 (C343), which is a fused-ring anion with the negative charge on the carboxylate group. Ground-state C343 has a small dipole moment, which increases in magnitude upon electronic excitation. ${ }^{14,52}$ In this initial computational study, we use a simplified model for the chromophore. The model, which in the $\mathrm{S}_{0}$ state corresponds to a rigid version of $\mathrm{I}_{2}{ }^{-53}$ retains the essentials of the $\mathrm{C} 343$ charge distribution and its change resulting from the $S_{0} \rightarrow S_{1}$ electronic transition: the solute has the charge of $-e$, which in the $S_{0}$ state is symmetrically distributed on the two I atoms, giving each the partial charge of $-e / 2$ and a zero dipole moment (measured from the molecular center-of-mass). The $S_{0} \rightarrow S_{1}$ transition is modeled as charge-localization on one of the I atoms: (-e/2,$e / 2) \rightarrow(0,-e)$. This transition leads to creation of a dipole moment of magnitude $e r_{I I} / 2=7.76 \mathrm{D}$, where $r_{I I}=3.23 \AA$ is the I-I bond length. The magnitude of this dipole is a little over three times larger than the $\mathrm{C} 343$ dipole change, estimated at $2.5 \mathrm{D},{ }^{52}$ although the size of our model chromophore is smaller. The potential parameters for the RM components and the solute are summarized in Table 1.

Integration of the MD trajectories was performed using the velocity Verlet algorithm with a 2 fs step size and appropriate bond constraints. ${ }^{54}$ The temperature was regulated using the Berendsen thermostat ${ }^{55}$ with a time constant of 0.4 ps during the equilibration periods and 2 ps during the production runs, which also included the nonequilibrium trajectories. Because these trajectories were only 2 ps long, however, the temperature regulation had little effect.

The RMs and HCs used in the present study were generated by adding an $\mathrm{I}_{2}^{-}$chromophore to the core region of the equilibrated structures generated in our earlier study ${ }^{11}$ and reequilibrating. In the $\mathrm{RMs}$ an additional $\mathrm{Na}^{+}$counterion was 
TABLE 1: Potential and Structural Parameters

\begin{tabular}{lccc}
\hline \multicolumn{3}{c}{ A. Lennard-Jones + coulomb potential parameters } \\
\hline \multicolumn{1}{c}{ species } & $\sigma / \AA$ & $(\epsilon / k) / \mathrm{K}$ & $q / e$ \\
\hline wall $^{a}$ & 2.500 & 231.55 & 0 \\
$\mathrm{O}$ & 3.166 & 78.24 & -0.8476 \\
$\mathrm{H}$ & 0 & 0 & 0.4238 \\
$\mathrm{Na}+$ & 2.275 & 58.01 & 1.0 \\
$\mathrm{Z}^{-}$(headgroups) & 6.000 & 251.58 & -1.0 \\
$\mathrm{I}(1) \mathrm{b}$ & 3.61 & 169.50 & $-0.5,0.0$ \\
$\mathrm{I}(2) \mathrm{b}$ & 3.61 & 169.50 & $-0.5,-1.0$ \\
\hline
\end{tabular}

B. molecular structure and harmonic potential parameters

water: $R_{\mathrm{OH}}=1.0 \AA, \angle_{\mathrm{HOH}}=109.5^{\circ} ; \mathrm{I}_{2}^{-}: R_{\mathrm{II}}=3.23 \AA$ headgroup-wall: $d_{e}=2.5 \AA, k_{e}=600 \mathrm{kcalmol}^{-} 1 \AA^{-2}$

${ }^{a}$ The functional form of the potential between the cavity and interior sites is given in ref $11 .^{b}$ The first set of partial charges is for the $S_{0}$ and the second for the $S_{1}$ state.

TABLE 2: Composition and Size of Reverse Micelles and Hydrophobic Cavities Used in SD Simulations ${ }^{a}$

\begin{tabular}{lcccc}
\hline system & $n\left(\mathrm{H}_{2} \mathrm{O}\right)$ & $n\left(\mathrm{Na}^{+}\right)$ & $n\left(\mathrm{Z}^{-}\right)$ & $R_{\text {cavity }}(\AA)$ \\
\hline$w_{0}=1$ & $21(21)$ & $22(21)$ & $21(21)$ & $10.33(10.30)$ \\
$w_{0}=2$ & $52(52)$ & $27(26)$ & $26(26)$ & $11.70(11.60)$ \\
$w_{0}=4$ & $140(140)$ & $36(35)$ & $35(35)$ & $14.05(14.10)$ \\
$w_{0}=7.5$ & $516(525)$ & $71(70)$ & $70(70)$ & $19.4(19.4)$ \\
HC_174 & $170(174)$ & 0 & 0 & $13.4(13.4)$ \\
HC_674 & $668(674)$ & 0 & 0 & $19.4(19.4)$
\end{tabular}

${ }^{a}$ The numbers in brackets refer to reverse micelles without the chromophore.

also added to maintain charge balance. In our previous simulations of RMs without a chromophore, ${ }^{11}$ the numbers of water and AOT molecules corresponding to a given $w_{0}$ were taken from estimates based on light scattering data by Eicke and Rehak $^{12}$ for RMs in which the oil phase is made up of isooctane. The RM radii were determined by assigning $w_{0}$-independent molecular volumes to water, $\mathrm{Na}^{+}$counterions, and sulfonate headgroups, and were checked against experimental estimates. ${ }^{12}$ Two different procedures were employed to account for the additional volume occupied by the chromophore. In the RMs with $w_{0}=1,2$, and 4 , following random placement of the chromophore, the cavity radius was adjusted assuming a volume of $60 \AA^{3}$ for $\mathrm{I}_{2}^{-}$. Although this procedure generally leads to a slight increase in the cavity radius, the radius of the $w_{0}=4$ RM was actually decreased slightly due to a small error in the previous value. With the second procedure, which was employed on the largest RM and the two HCs, the cavity radius was held fixed, the chromophore was placed randomly within the core region, and overlapping water molecules were removed. With both procedures, many placements were attempted in order to minimize highly repulsive chromophore-solvent interactions or the number of removed solvent molecules. The main advantage of the first procedure is that the water-to-surfactant ratio is not changed by the addition of the chromophore, while the drawback is that a long period of reequilibration is required. Configurations produced by the second placement method required shorter reequilibration times, but tend to lose more than the $2-3$ water molecules that the additional volume of the chromophore would seem to require. Table 2 lists the radii and contents both before and after the addition of the chromophore for all of the systems studied here.

After placement of the chromophore, the systems were reequilibrated for 50-2000 ps, depending on the size of the system and the chromophore placement method employed. As we show later, $\mathrm{I}_{2}{ }^{-}$is located predominantly in the $\mathrm{RM}$ core region. To make sure this location is unrelated to the starting configuration, we also equilibrated the $w_{0}=4 \mathrm{RM}$ with $\mathrm{I}_{2}{ }^{-}$ tethered to the wall. Upon release from the tether, $\mathrm{I}_{2}{ }^{-}$ended up in the core region, with the same distance distribution relative to the interface obtained when it is initially placed in the core region.

II.B. Computer Simulation of Solvation Dynamics. SD refers to the rate of solvent reorganization in response to a change in solute properties brought about by its electronic excitation. ${ }^{56-60,13}$ This response is usually measured as a timedependent Stokes shift in the fluorescence spectrum of the solute. Its time-evolution is characterized in terms of the normalized response $\mathrm{e}^{60,13}$

$$
S(t)=\frac{v_{\max }(t)-v_{\max }(\infty)}{v_{\max }(0)-v_{\max }(\infty)}
$$

of the peak frequency $v_{\max }$ of the spectrum at time $t$ after excitation, which occurs at $t=0 . v_{\max }(\infty)$ denotes the steadystate emission peak frequency. The time-evolution of the Stokes shift is due to the solvent effects on the vertical energy gap between the $\mathrm{S}_{0}$ and $\mathrm{S}_{1}$ solute electronic states. C343 and other chromophores that are used in SD experiments are structurally quite rigid, and their structure is weakly perturbed by the $S_{0} \rightarrow$ $\mathrm{S}_{1}$ electronic transition, so the main source of the solvent effect is the change $\Delta E$ in the solute-solvent potential ${ }^{60,13}$

$$
\Delta E=U_{1}-U_{0}
$$

where $U_{n}$ is the solute-solvent potential in the solute electronic state $S_{n}$. To express $S(t)$ in terms of $\Delta E$, one relates it to $v_{\max }$ by $^{61}$

$$
h v_{\max }(t)=\overline{\Delta E(t)}+h v_{\mathrm{el}}
$$

where $v_{\mathrm{el}}$ is the electronic transition frequency for the isolated solute and the overbar indicates an average over different microscopic solvent environments corresponding to the macroscopic experimental conditions: the solvent is initially equilibrated with the ground-state solute and the solute-solvent potential is instantaneously changed at $t=0$. Using eq 6 , the solvation response becomes

$$
S(t)=\frac{\overline{\Delta E(t)}-\overline{\Delta E(\infty)}}{\overline{\Delta E(0)}-\overline{\Delta E(\infty)}}
$$

Although there may be some changes in the nonelectrostatic portion of the solute-solvent potential, most of $\Delta E$ results from the change in the charge distribution of the chromophore. In our model the LJ portion of the potential does not depend on the electronic state of the chromophore, so $\Delta E$ is purely electrostatic

$$
\Delta E=\sum_{j=1}^{N} \sum_{\alpha \in 0} \sum_{\beta \in j} \frac{\Delta q_{0 \alpha} q_{j \beta}}{4 \pi \epsilon_{0}\left|\mathbf{r}_{0 \alpha}-\mathbf{r}_{j \beta}\right|}
$$

where $j$ denotes the $j$ th solvent molecule, $\alpha$ denotes a solute (molecule 0) site, $\beta$ a site on the $j$ th solvent molecule, $\Delta q_{0 \alpha}$ the change due to electronic excitation in the partial charge of the solute site $\alpha, \mathbf{r}_{0 \alpha}$ the position of this site, $q_{j \beta}$ the partial charge on site $\beta$ on the $j$ th solvent molecule and $\mathbf{r}_{j \beta}$ the position of this site.

As noted above, the model chromophore used in our simulations, rigid $\mathrm{I}_{2}{ }^{-}$, was chosen to resemble $\mathrm{C} 343$ in the values of its two lowest electrical multipoles in the $S_{0}$ and $S_{1}$ states. 
TABLE 3: Information on Molecular Dynamics Trajectories

\begin{tabular}{lccc}
\hline system & $\begin{array}{c}\text { number of } \\
\text { nonequilibrium } \\
\text { trajectories }\end{array}$ & $\begin{array}{c}\mathrm{S}_{0} \text { state } \\
\text { equilibrium } \\
\text { trajectory }(\mathrm{ps})\end{array}$ & $\begin{array}{c}\mathrm{S}_{1} \text { state } \\
\text { equilibrium } \\
\text { trajectory }(\mathrm{ps})\end{array}$ \\
\hline$w_{0}=1$ & 500 & 2000 & 2000 \\
$w_{0}=2$ & 500 & 2000 & 2000 \\
$w_{0}=4$ & 500 & 2000 & 2000 \\
$w_{0}=7.5$ & 300 & 1000 & 400 \\
HC_174 & 400 & 2000 & 400 \\
HC_674 & 200 & 1000 & 400
\end{tabular}

In a medium such as the RM interior, containing a highly polar liquid and ionic species, the response to a change in the solute dipole is expected to be highly collective, with a significant portion of $S(t)$ arising from molecules that are not in direct contact with the solute. Thus, we expect that our simple model probe will produce an $S(t)$ in reasonable agreement with experiment.

To simulate SD, we have carried out both equilibrium and nonequilibrium MD simulations with solute-containing RMs and hydrophobic cavities. Equilibrium trajectories with the solute in $\mathrm{S}_{0}$ were used to generate starting configurations, sampled at equal time intervals, for the nonequilibrium trajectories and to compute equilibrium time correlation functions (TCFs). Equilibrium trajectories with the solute in $\mathrm{S}_{1}$ were used to determine $\overline{\Delta E(\infty)}$. In the nonequilibrium trajectories, the solute charges were changed instantaneously in the starting configuration from the $S_{0}$ distribution to the $S_{1}$ distribution, and each trajectory was integrated for $2 \mathrm{ps}$ in order to compute $\overline{\Delta E(t)}$, the timedependent Stokes shift. Between 200 and 2500 trajectories were run for each system. The number of nonequilibrium trajectories and the lengths of the equilibrium runs are given in Table 3.

The equilibrium trajectory data were used to generate the TCFs

$$
C_{0}(t)=\langle\delta \Delta E(0) \delta \Delta E(t)\rangle_{0} /\left\langle[\delta \Delta E]^{2}\right\rangle_{0}
$$

of fluctuations $\delta \Delta E=\Delta E-\langle\Delta E\rangle_{0}$ in $\Delta E$, where $\langle\cdots\rangle$ denotes an equilibrium ensemble average and the subscript 0 indicates that it was evaluated in the presence of the $S_{0}$ solute. One can define in an analogous way $C_{1}(t)$, the TCF of $\delta \Delta E$ in the presence of the $S_{1}$ solute. Within the stationary linear response approximation, in which the response is assumed independent of the time origin ${ }^{62}$

$$
S(t) \cong C_{0}(t) \cong C_{1}(t)
$$

As we shall see, the approximation $S(t) \cong C_{0}(t)$ is reasonably good in the present case, especially for the longer-time response, and we can therefore use the analysis of $C_{0}(t)$ to investigate the role of intermolecular correlations to $\mathrm{SD}$ in these confined aqueous systems.

\section{Results}

III.A. Basic Structural Features of Reverse Micelles Containing the Model Chromophore. In our previous study of the RM interior, ${ }^{11}$ we found that the basic structural and dynamical properties of water and counterion molecules were strongly dependent on their distance from the surfactant interface, defined by the average position of headgroups. For the model used here, this interface is simply a sphere of radius $2.5 \AA$ less than the full cavity. To characterize the structure of the chromophore-containing cavities, we have computed the densities of the molecular components as a function of the distance from the interface.

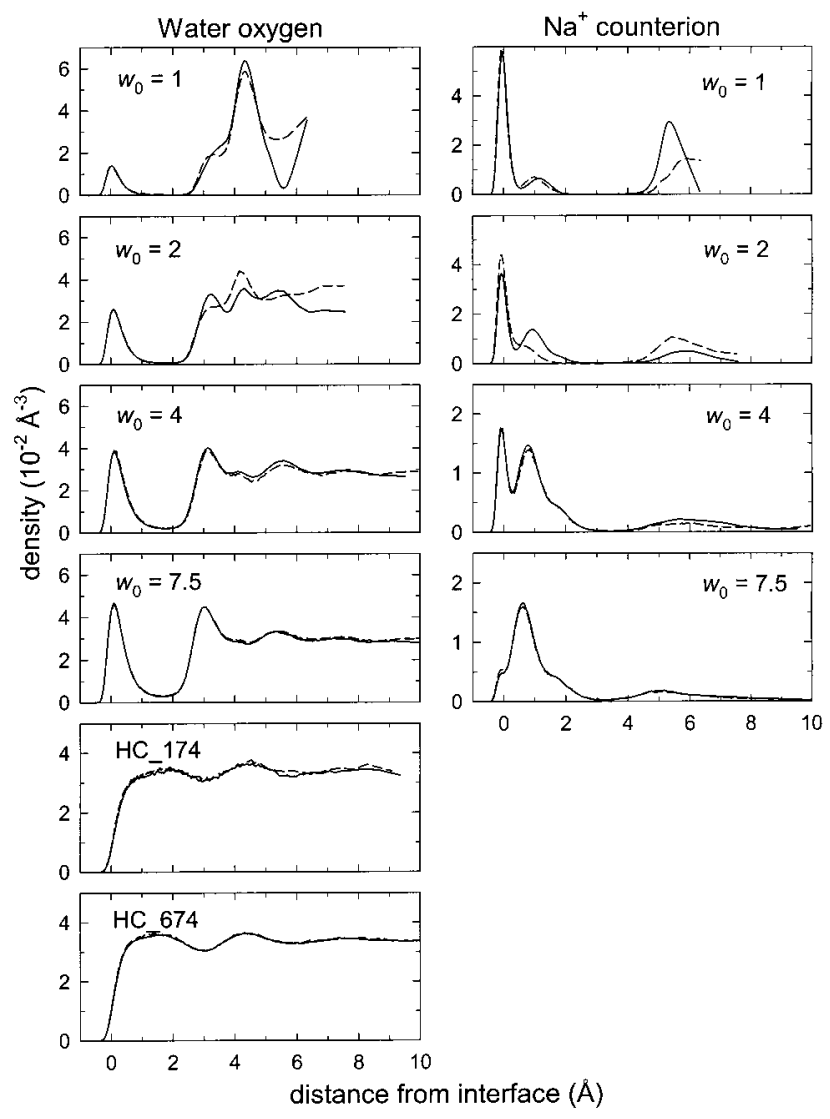

Figure 1. Average densities as a function of distance from the cavity interface for water oxygens and $\mathrm{Na}^{+}$counterions in systems with (solid lines) and without (dashed lines) the $\mathrm{I}_{2}{ }^{-}$solute chromophore. The cavity interface is defined as a surface $2.5 \AA$ inside the cavity boundary that marks the equilibrium location of the headgroup ions and the edge of the repulsive region for molecule-cavity interactions.

Figure 1 shows density profiles for water oxygen atoms and $\mathrm{Na}^{+}$counterions as functions of distance from the interface for all of the systems studied, both containing and not containing the chromophore. The basic layered structure of water on the interior of both RMs and HCs is only minimally affected by the presence of the additional solute, and the effect diminishes with increasing cavity size. In our previous study, we labeled three distinct water regions within the RMs based on these density profiles. The trapped water region encompasses the first peak in the water density, which is centered near a distance of $0 \AA$. These water molecules are literally trapped between the headgroup anions and exhibit very long residence times and greatly reduced mobilities (order of magnitude or more), both translational and rotational. The layer of bound water is identified with the second and third water peaks, centered at about 3 and $4 \AA$, respectively, and extends to between 4.5 and $5 \AA$. Water in this region still interacts strongly with the interface and is highly structured, but exhibits mobilities that are reduced by a factor of $2-3$ from bulk. Beyond the bound layer, water mobility increases further and its structural characteristicsnumber of hydrogen bonds and nearest neighbor interactionsapproach those of the bulk liquid. In the RMs, chromophore addition has almost no effect on the density profiles of trapped water, which suggests that the solute has a minimal effect on the structure of this layer. In the two smaller RMs, the intensities of the bound water peaks do change somewhat, but the structure of the interior is not fundamentally altered. The preservation of interior structure is also reflected in the counterion density profiles, which change significantly only for $w_{0}=2$. As we 

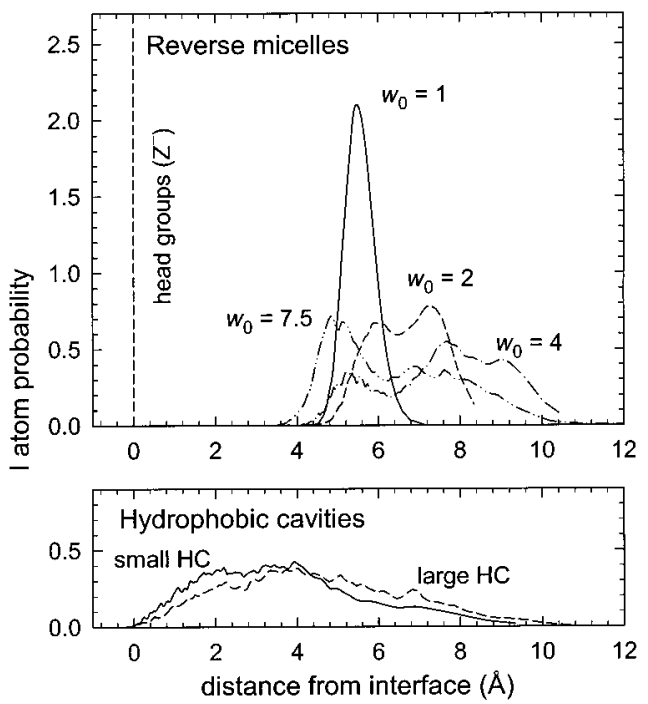

Figure 2. Solute I atom probability densities for all systems studied as a function of the distance from the cavity interface.

discuss in more detail below, the small changes that do occur to both the water and counterion density profiles reflect the location of the chromophore predominantly in the core region of the RMs.

The surface area per headgroup increases with $w_{0}$ from 36 $\AA^{2}$ to $51 \AA^{2}$ over the range considered here. ${ }^{12,11}$ This is reflected by the increase in the 'trapped' water peak with increasing $w_{0}$ of the water density profile as more space between headgroups becomes available. In contrast to the RMs, the $\mathrm{HC}$ water density profiles show relatively little structuring, their most prominent feature being the density depletion near the hydrophobic wall. The $\mathrm{Na}^{+}$ions in the RMs are mainly in the interfacial region. In the smaller RMs, the density profile exhibits a sharp peak at $0 \AA$, indicating that $\mathrm{Na}^{+}$ions, which are much smaller than $\mathrm{Z}^{-}$, pack into the voids among closely packed headgroups. The size of this peak decreases with $w_{0}$ as the size of the water oxygen peak increases. We have found that, as the RMs get larger, $\mathrm{Na}^{+}$ions coordinate a larger number water molecules and fewer headgroup ions, allowing them to penetrate further into the RM interior, as shown in the spreading of the $\mathrm{Na}^{+}$ density profile.

Figure 2 shows the probability densities, normalized per unit of distance, for finding a solute I atom at a given distance from the surfactant interface. The most important feature of these profiles in terms of its implications for solvation dynamics is the complete exclusion of the chromophore from the interfacial layer in the RMs. The large size of the chromophore and its negative charge appear to preclude any penetration of the interfacial region in the RMs, which is dominated by the large headgroup anions. In the $\mathrm{HCs}$, on the other hand, the chromophore resides preferentially near the surface, although the distribution is very broad, suggesting relatively free motion of the solute inside these cavities. In the core region of the RMs, the chromophore interacts primarily with bound and free water molecules, which have properties that are relatively similar to those of the bulk in comparison to the trapped water. This suggests that the magnitude and time scale for the solvation response will be more similar to the bulk than if the chromophore were in greater contact with the interface. The trends in the solute atom distributions in the RMs can be explained by two main factors: size of the core region and density of surfactant headgroups at the interface. In the smallest RM, $w_{0}$ $=1$, the small cavity size confines the chromophore to a
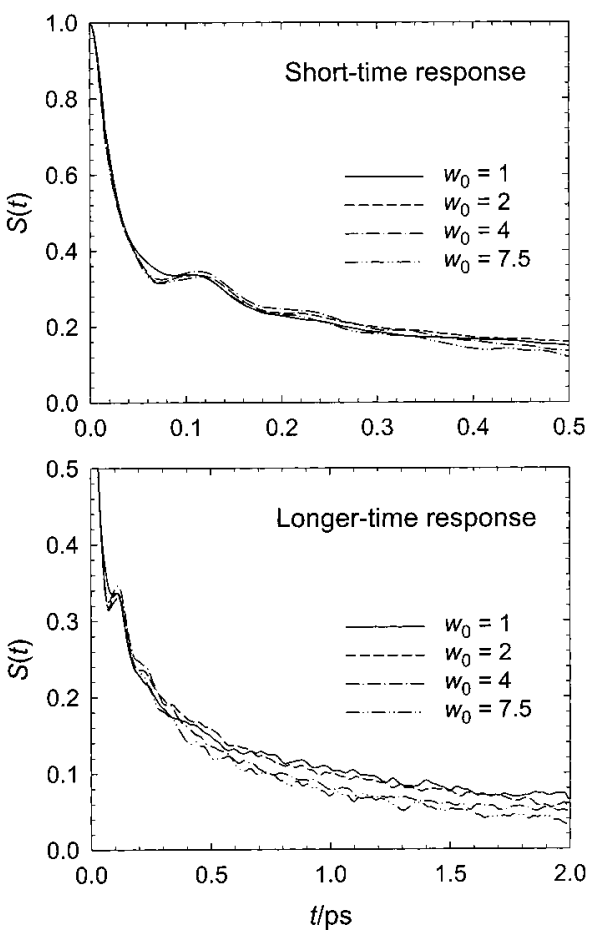

Figure 3. Solvation response, $S(t)$, in reverse micelles of varying size ranging from $w_{0}=1$ to $w_{0}=7.5$. The top panel depicts the short-time response and the bottom panel the longer-time response. Note that the lower panel covers only the $0.0-0.5$ range of variation of $S(t)$.

relatively narrow range of distances. As the cavity size increases, the distribution becomes broader and bimodal. The outer peak of this distribution reflects the attachment of at least one solute atom to the bound water layer a substantial fraction of the time. The inner peak may reflect alignment of the bound chromophores, whereas the broad distributions in the core region suggest that the chromophore moves relatively freely in the core for a significant fraction of the time. The distributions also exhibit greater penetration of the chromophore into the bound water region with increasing RM size, which probably results from the decrease in surface ion density and the accompanying increase in the flexibility of the bound water layer.

III. B. Solvation Dynamics-Nonequilibrium Response. We turn now to SD in RMs in order to determine how the properties of this heterogeneous and confined environment affect $S(t)$. Figure 3 depicts our results for $S(t)$ in RMs of varying water content. The top panel illustrates the short-time response for 0 $\leq t \leq 0.5 \mathrm{ps}$ and the bottom panel focuses on the longer-time decay, depicting the interval $0 \leq t \leq 2 \mathrm{ps}$ and the values of $S(t)$ $\leq 0.5$.

The most striking feature of our $S(t)$ results is that they are relatively insensitive to the RM size. The solvation responses for RMs in the $w_{0}$ range of $1-7.5$, exhibit nearly identical the initial inertial decay rates and hydrogen $(\mathrm{H})$ bond librational features in the $0.02 \mathrm{ps} \leq t \leq 0.20 \mathrm{ps}$ time interval. The $w_{0}$ dependence of $S(t)$ becomes significant only at times longer than about 0.2 ps. However, by this time $S(t)$ has decayed to about $25 \%$ of its initial value. For the smallest RM, $w_{0}=1$, the amplitude of librational oscillations is reduced, but the decay rate actually exceeds that of the $w_{0}=2$ and 4 RMs for several hundred fs.

On the longer time scale displayed in the bottom panel of Figure 3, the differences in the decay rates of $S(t)$ for different size RMs are more evident, with the larger RMs exhibiting a faster decay over the $0.5-2.0 \mathrm{ps}$ time scale. However, it is worth stressing that this time scale difference concerns a small portion 

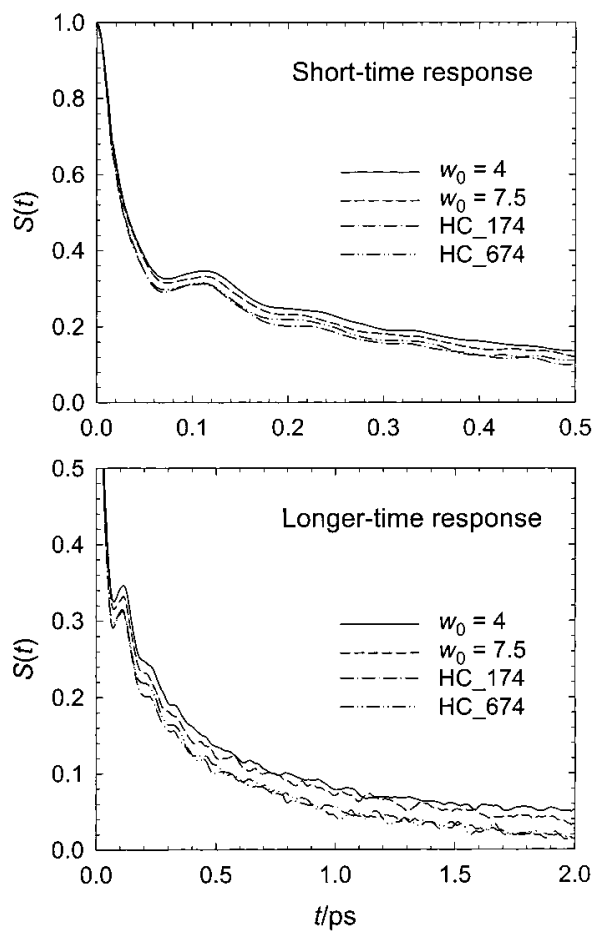

Figure 4. Comparison of the solvation responses in the reverse micelles corresponding to $w_{0}=4.0$ and 7.5 and in similar-size hydrophobic cavities, HC_174 and HC_674. The top panel depicts the short-time response and the bottom panel the longer-time response.

of the overall decay of $S(t)$, since $85-90 \%$ of the decay occurs at shorter times for which the response in only weakly dependent on RM size.

The very fast initial decay of $S(t)$ is well-known to occur in bulk water ${ }^{17,16,18-24,14,15}$ and can be explained in terms of highly collective response, ${ }^{17,16}$ small moments of inertia of water molecules, and high-frequency librational dynamics. ${ }^{20,21}$ Our results show that much of $S(t)$ in RMs decays on a similar scale, suggesting that water, and not the ions, accounts for the solvent response in this regime and further that the water response on this time scale is not sensitive to the structural and dynamical heterogeneity of these systems. The location of this particular probe molecule in the core region of the RMs accounts for some of this insensitivity, but our previous simulations demonstrated a significant decrease in water mobilities in the core regions of the smaller RMs. We therefore would have expected to see a larger $w_{0}$-dependence in $S(t)$.

The influence of water on the overall response will be gauged in two ways: First, by comparing the $S(t)$ 's in RMs and in HCs, and, second, by decomposing the total $S(t)$ in RMs into the responses of the three solvent components. This can be done because our $\Delta E$, eq 8 , is pairwise-additive, which leads to

$$
S(t)=S_{\mathrm{H}_{2} \mathrm{O}}(t)+S_{\mathrm{Na}^{+}}(t)+S_{\mathrm{Z}^{-}}(t)
$$

where $S_{m}(t)$, the response of component $m$, is given by

$$
S_{m}(t)=\frac{\overline{\Delta E_{m}(t)}-\overline{\Delta E_{m}(\infty)}}{\overline{\Delta E(0)}-\overline{\Delta E(\infty)}}
$$

Figure 4 shows a comparison in the solvation responses in RMs and HCs of similar size. As can be seen from Table 2, the radii of the $w_{0}=4$ and 7.5 RMs are similar to those of HC_174 and HC_674, respectively. As in Figure 3, the top panel illustrates the short-time and the bottom panel the longer-time
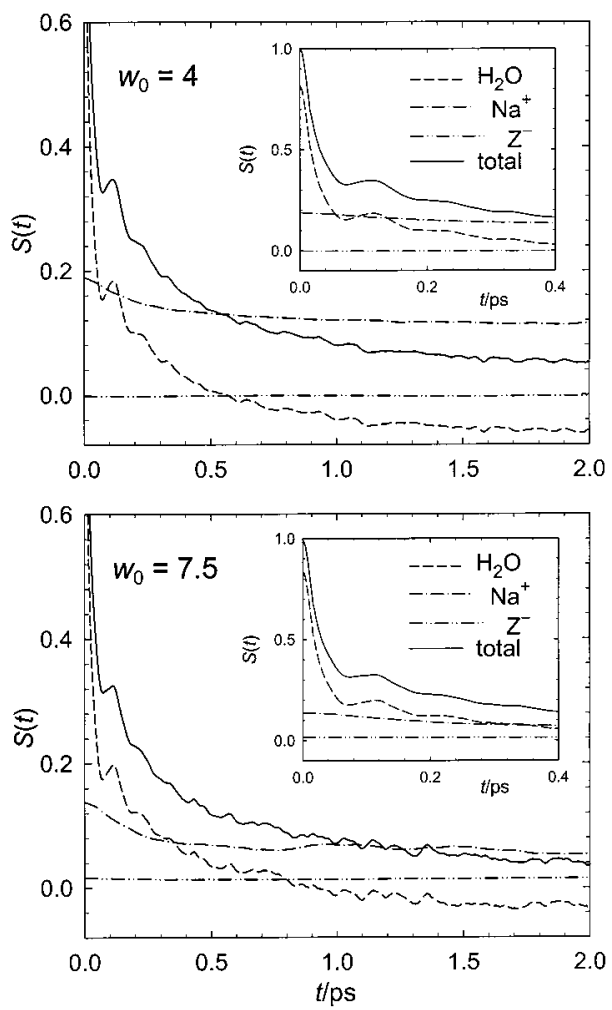

Figure 5. Decomposition of the solvation response, $S(t)$, in the $w_{0}=$ 4.0 and 7.5 reverse micelles into contributions from different solvent components: water, the $\mathrm{Na}^{+}$counterions, and the surfactant headgroups.

response. The results depicted in Figure 4 show that $S(t)$ decays faster in $\mathrm{HCs}$ than in RMs of similar size, but that the difference in the decay rates is quite small in the $0-0.03$ ps inertial regime and that the librational features occurring over the $0.03-0.20$ ps time scale have similar oscillation periods. Their amplitudes are a little higher in the $\mathrm{HCs}$, consistent with the fact that these systems have a larger number of water-water $\mathrm{H}$-bonds per molecule. ${ }^{11}$ The differences in the diffusive portion of the decay $(t>0.20 \mathrm{ps})$ are more pronounced. Furthermore, while this portion of $S(t)$ in HCs is nearly independent of the cavity size, its decay rate clearly increases with size in the RMs. This is consistent with the nearly identical solute atom distribution profiles (see Figure 2) in the two HCs and the fact that water mobilities within the HCs exhibit no size-dependence. ${ }^{11}$

The similarity between the short-time responses in HCs and RMs strongly suggests that water is the main contributor to this part of the response in the case of RMs. The differences seen at longer times may be due to the reduced water mobility in RMs, especially at lower $w_{0}$ values, and to the participation of the headgroups and counterions to the response. To clarify these issues, we display in Figure 5 the decomposition of $S(t)$ in the $w_{0}=4$ and 7.5 RMs into contributions from different solvent species. In both of these RMs, as well as in the two RMs for which the results are not displayed, the headgroups account for a very small portion of $S(t)$. This is not surprising in view of the fact that the headgroups are much less mobile than other solvent species, given their large mass and the fact that they are harmonically bound to the interface in the radial direction. The short-time dynamics of solvation is dominated by water, as the comparison with the $\mathrm{HC}$ results suggested. At longer times, the $\mathrm{Na}^{+}$ions make a significant contribution to $S(t)$. This response appears to be anticorrelated with that of water. In both RMs, the longer-time $S_{\mathrm{H}_{2} \mathrm{O}}(t)$ is negative and $S_{\mathrm{Na}^{+}}(t)$ positive. This indicates that, due to its faster inertial response, water initially oversolvates the excited-state solute and subsequent 
TABLE 4: Steady-state Fluorescence Stokes Shift Values

\begin{tabular}{|c|c|c|c|c|c|}
\hline \multirow[b]{2}{*}{ system } & \multicolumn{4}{|c|}{$\frac{\overline{\Delta E(0)}-\overline{\Delta E(\infty)}}{h c} / 10^{4} \mathrm{~cm}^{-1}$} & \multirow{2}{*}{$\begin{array}{c}\left\langle(\delta \Delta E)^{2}\right\rangle_{0} \\
h c k T \\
10^{4} \mathrm{~cm}^{-1}\end{array}$} \\
\hline & $\mathrm{H}_{2} \mathrm{O}$ & $\mathrm{Na}^{+}$ & $\mathrm{Z}^{-}$ & total & \\
\hline$w_{0}=1$ & 2.43 & 0.64 & 0.01 & 3.06 & 3.84 \\
\hline$w_{0}=2$ & 2.35 & 0.78 & -0.03 & 3.10 & 3.72 \\
\hline$w_{0}=4$ & 2.52 & 0.56 & -0.02 & 3.06 & 3.62 \\
\hline$w_{0}=7.5$ & 2.63 & 0.37 & 0.06 & 3.07 & 3.67 \\
\hline HC_174 & 3.03 & & & 3.03 & 4.21 \\
\hline $\mathrm{HC}^{-} 674$ & 3.03 & & & 3.03 & 4.24 \\
\hline
\end{tabular}

solvent reorganization includes replacement of water by $\mathrm{Na}^{+}$ in the nearby solvation shells. Similar steps in longer-time solvation dynamics have been observed in polar solvent mixtures ${ }^{63,64}$ and electrolyte solutions. ${ }^{65}$

The contributions of each of the solvent components to $\overline{\Delta E(0)}-\overline{\Delta E(\infty)}$, the steady-state Stokes shift, in the solute fluorescence spectrum, are given in Table 4. The total shift is quite insensitive to RM size, but the contributions from the water and counterions vary substantially.

In general, the contribution of $\mathrm{Na}^{+}$decreases and the contribution of water increases with increasing $w_{0}$, as one might have anticipated based on the smaller ionic concentrations in the larger RMs, although this trend is reversed in going from $w_{0}=1$ to $w_{0}=2$. Extrapolation of the experimental results for $\overline{\Delta E(t)}-\overline{\Delta E(\infty)}$ for $\mathrm{C} 343$ in water/AOT/isooctane to $t=0,{ }^{40}$ using the method described by Fee and Maroncelli, ${ }^{41}$ also yields values of the steady-state Stokes shift that are weakly dependent on $w_{0}$. However, these values are about a factor of 15 smaller than the ones obtained in our simulation. This difference is due to the larger dipole change on electronic excitation and the smaller size of $\mathrm{I}_{2}{ }^{-}$relative to the $\mathrm{C} 343$ chromophore used in experiments. We are presently carrying out SD simulations using a model chromophore that resembles more closely C343.

In addition to examining how different solvent components contribute to $\mathrm{SD}$, it is instructive to decompose $S(t)$ into contributions from the two solute interaction sites. Site 1 undergoes partial charge extinction: $-e / 2 \rightarrow 0$, whereas site 2 undergoes an augmentation in the magnitude of its negative charge: $-e / 2 \rightarrow-e$. Again, because $\Delta E$ is a sum of site-site Coulomb potentials, $\Delta E=\Delta E_{1}+\Delta E_{2}$, and

$$
S(t)=S_{1}(t)+S_{2}(t)
$$

Figure 6 depicts these two components for two RMs, $w_{0}=1$ and 4, and the larger of the two hydrophobic cavities, HC_674. It shows that most of the inertial decay of $S(t)$ can be ascribed to $S_{1}(t)$ and all of the librational and diffusive portions to $S_{2}(t)$. $S_{1}(t)$ decays rapidly to zero on a time scale that is remarkably similar in the three systems. This decay is due to the inertial response of water and most likely corresponds to the motion of water hydrogen atoms away from site 1 . This is the mechanism that causes the short-time response to charge migration in an anion-neutral pair in methanol. ${ }^{66}$ The response of site 1 does not extend beyond the inertial time scale because the site becomes uncharged and thus does not experience any electrostatic forces capable of changing $\Delta E$. The differences among the systems can be attributed mainly to $S_{2}(t)$, which is affected by the variations in water-water hydrogen bond densities in the vicinity of the solute, the reduced mobility of water in the RMs, and the motion of the $\mathrm{Na}^{+}$ions to solvate the larger negative charge acquired by site 2 upon electronic excitation. The hydrogen bond densities, which are greatly reduced in the smaller RMs (see Figure 8 of ref 11), influence the amplitudes

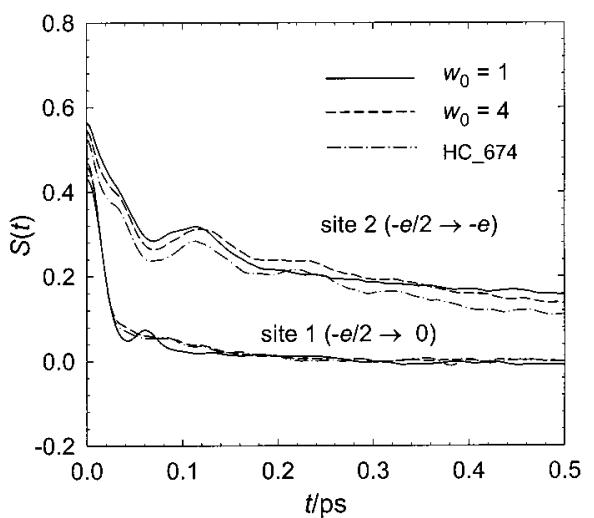

Figure 6. Decomposition of the solvation response, $S(t)$, into contributions $S_{1}(t)$ and $S_{2}(t)$ from the two solute sites: site 1 which undergoes charge extinction, $-e / 2 \rightarrow 0$, and site two which undergoes an increase in negative charge, $-e / 2 \rightarrow-e$. Depicted are the results for the reverse micelles corresponding to $w_{0}=1.0$ and 4.0 and for the hydrophobic cavity, HC_174.
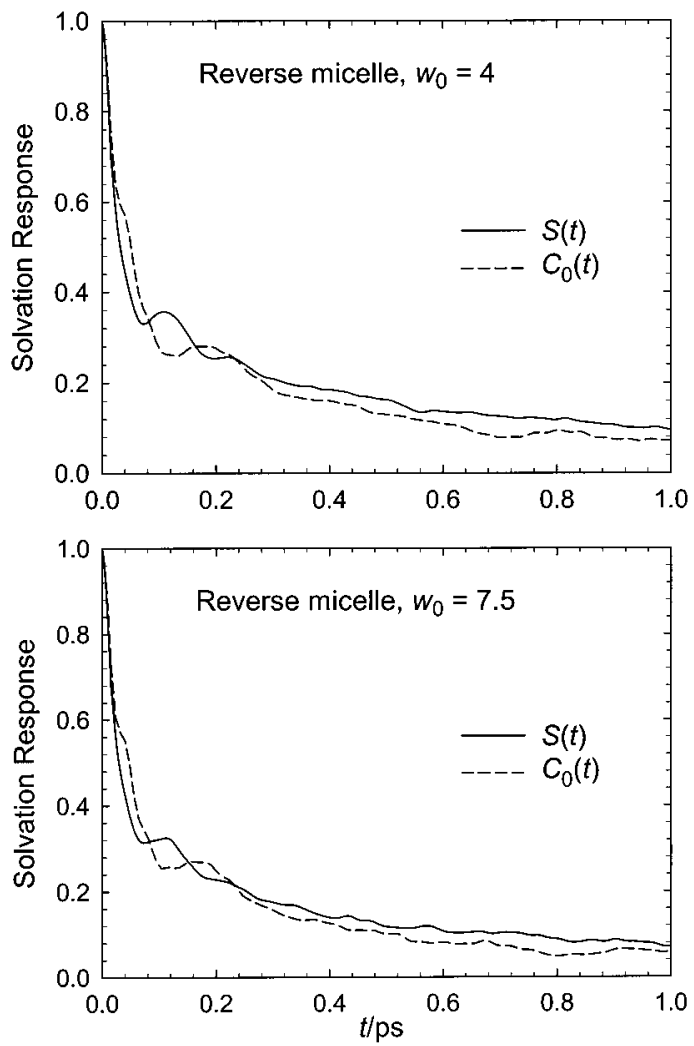

Figure 7. Comparison of the solvation response, $S(t)$, and the time correlation, $C_{0}(t)$, of fluctuations in $\Delta E$ for the solvent in equilibrium with the ground-state solute for the reverse micelles $w_{0}=4$ (top panel) and $w_{0}=7.5$ (bottom panel).

of librational oscillations in $S(t)$, whereas the reduced water mobility and the counterion response (see Figure 5) in the RMs lead to a slower diffusive response.

III. C. Solvation Dynamics-Solvation Time Correlations. Analysis of the TCF of fluctuations in $\Delta E$ can provide helpful information about the SD mechanism if the linear response approximation (LRA) connecting $S(t)$ and $C_{0}(t)$ is reasonably accurate. We therefore start by testing this approximation for the RMs and HCs of interest here. Some of the results of this test are shown in Figures 7 and 8. Figure 7 depicts the $S(t)-$ $C_{0}(t)$ comparison for the RMs corresponding to the water content values $w_{0}=4$ and 7.5, whereas Figure 8 displays the 

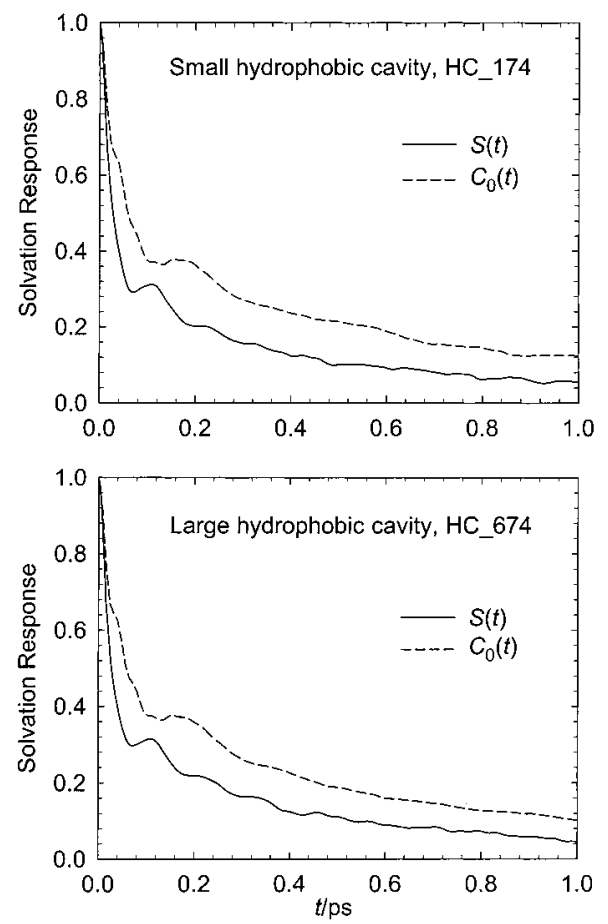

Figure 8. Same as Figure 8, but for solvation dynamics in hydrophobic cavities, HC_174 (top panel) and HC_674 (bottom panel).

corresponding data for the two HCs. Figure 7 indicates that the linear response approximation captures the overall time scale for the decay of $S(t)$ in the two RMs, but does not accurately describe the librational contribution to the solvation response on a sub-200 fs time scale. In the case of HCs, the overall agreement between $C_{0}(t)$ and $S(t)$ is still reasonable, but not as good, with $C_{0}(t)$ showing a larger amplitude diffusive component than $S(t)$ in addition to the discrepancies in the short time response.

The fact that $C_{0}(t)$ predicts an earlier onset of librational contributions to solvation dynamics can be understood by considering the results shown in Figure 6. Those results indicated that much of the early decay of $S(t)$ was due to the fast inertial dynamics of water in response to the charge extinction on solute site 1 . Given that $C_{0}(t)$ is calculated with site 1 charged, the surrounding water hydrogens experience electrostatic attraction to this site, which, in turn, couples $\Delta E_{1}$ fluctuations to hydrogen bond librations. Once this electrostatic attraction is turned off, rotation of hydrogens away from this site is not subject to the restoring forces of the hydrogen bond network. Despite these differences in the extent of coupling to water libration between $S(t)$ and $C_{0}(t)$, the LRA is reasonably accurate, especially for the RMs, and we can develop additional insights into the solvation mechanism by analyzing $C_{0}(t)$.

The extent of agreement with the LRA can be also tested for the steady-state Stokes shift. Within this approximation ${ }^{61}$

$$
\overline{\Delta E(0)}-\overline{\Delta E(\infty)} \cong \frac{\left\langle(\delta \Delta E)^{2}\right\rangle_{0}}{k T}
$$

An analogous relation would hold for the fluctuations in $\Delta E$ for the system containing the $S_{1}$ solute. Table 4 shows a comparison between the linear response estimate, eq 14, and the steady-state shifts observed in the simulations. The LRA always overestimates the steady-state Stokes shift-by 20-25\% for the RMs and by about $40 \%$ for the two HCs. These results
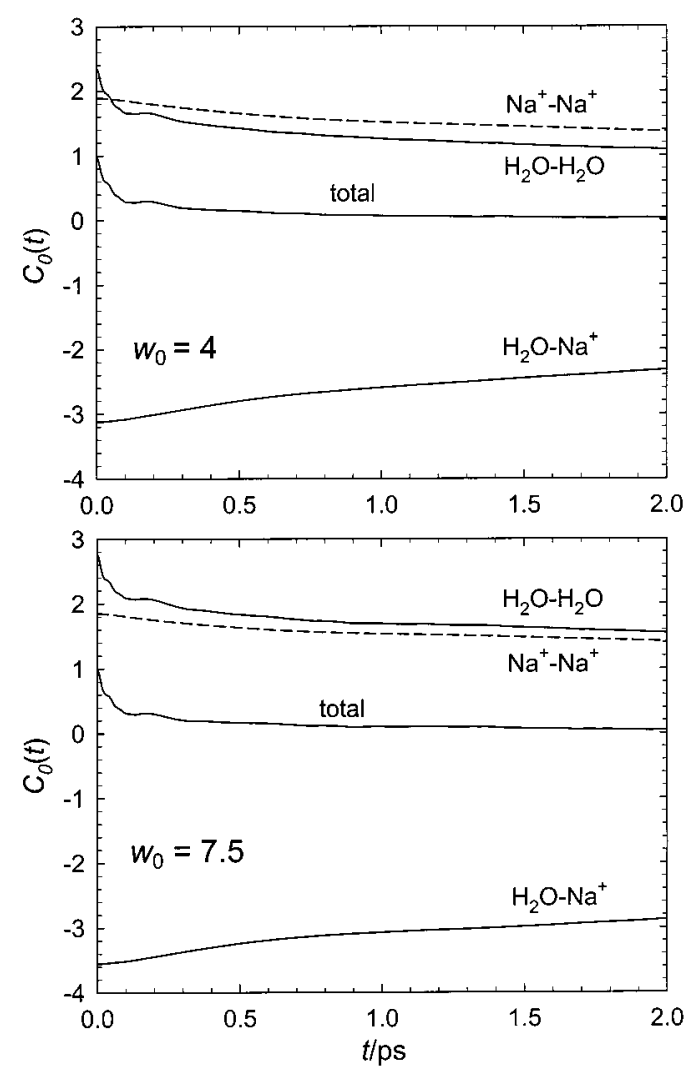

Figure 9. Decomposition of the ground-state solvation time correlation, $C_{0}(t)$, into contributions from the major solvent components, water and $\mathrm{Na}^{+}$counterions, for the reverse micelles $w_{0}=4$ (top panel) and $w_{0}=$ 7.5 (bottom panel). Depicted are the $\mathrm{H}_{2} \mathrm{O}-\mathrm{H}_{2} \mathrm{O}$ and $\mathrm{Na}^{+}-\mathrm{Na}^{+}$ autocorrelations and the $\mathrm{H}_{2} \mathrm{O}-\mathrm{Na}^{+}$cross-correlation.

are consistent with the extent of disagreement between $S(t)$ and $C_{0}(t)$, which is also larger for HCs than for RMs.

We start with the decomposition of $C_{0}(t)$ in $\mathrm{RMs}$ into contributions from the different solvent components

$$
\begin{array}{r}
C_{0}(t)=C_{0, \mathrm{H}_{2} \mathrm{O}-\mathrm{H}_{2} \mathrm{O}}(t)+C_{0, \mathrm{Na}^{+-} \mathrm{Na}^{+}}(t)+C_{0, \mathrm{Na}^{+-} \mathrm{H}_{2} \mathrm{O}}(t)+ \\
C_{0, \mathrm{H}_{2} \mathrm{O}-\mathrm{Z}^{-}}(t)+C_{0, \mathrm{Na}^{+-} \mathrm{Z}^{-}}(t)+C_{0, \mathrm{Z}^{--} \mathrm{Z}^{-}}(t)
\end{array}
$$

The decomposition of $S(t)$ in Figure 5 showed that only the motions of water and counterions make a significant contribution to the nonequilibrium solvation response. For $C_{0}(t)$ the major contributions come from the auto- and cross-correlations of $\delta \Delta E_{\mathrm{H}_{2} \mathrm{O}}$ and $\delta \Delta E_{\mathrm{Na}^{+}}$, given in the first line of eq 15 . Only these contributions are shown in Figure 9 for RMs with $w_{0}=$ 4 and 7.5. Additional contributions to $C_{0}(t)$ involving $\delta \Delta E_{\mathrm{Z}^{-}}$ are quite small and are therefore not displayed. Figure 9 shows that the water and counterion diffusive motions contributing to $\mathrm{SD}$ are strongly coupled and anticorrelated because the $\mathrm{Na}^{+}-$ $\mathrm{H}_{2} \mathrm{O}$ cross-correlation is large and negative. The rapid decay of $C_{0}(t)$ arises from cancellation of the positive diffusive portions of the two autocorrelations by the negative cross-correlation. The extent of cancellation is slightly larger in the case of the $w_{0}=7.5 \mathrm{RM}$, indicating that there are stronger $\mathrm{Na}+-\mathrm{H}_{2} \mathrm{O}$ cross-correlations present in this system than in the smaller RM, $w_{0}=4$. This may be explained by noting that the number of water molecules in the first coordination shell of $\mathrm{Na}^{+}$increases with $w_{0}$ over this range of RM sizes as the ion density decreases. ${ }^{11}$ The mobility of counterions between the headgroups also increases. 


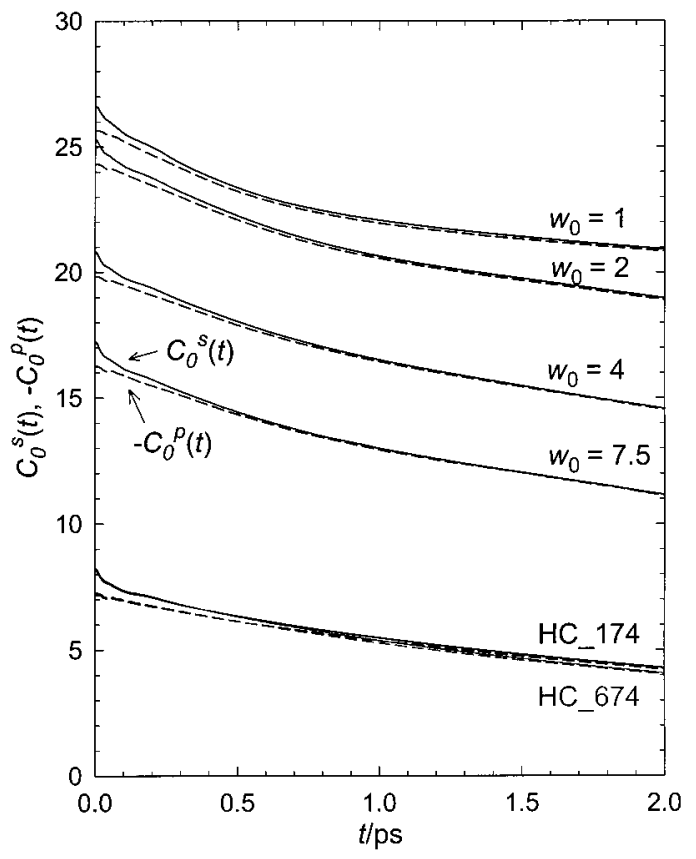

Figure 10. Decomposition of the ground-state solvation time correlation, $C_{0}(t)$, into single-solvent-molecule, $C_{0}^{s}(t)$, and solvent-pair, $C_{0}^{p}(t)$, contributions. Depicted are $C_{0}^{s}(t)$ (full line) and $-C_{0}^{p}(t)$, the negative of the solvent-pair component, (dashed line) for the reverse micelles $w_{0}$ $=1,2,4$, and 7.5 and for the two hydrophobic cavities HC_174 (black) and HC_674 (gray).

A useful way to assess the collective nature of SD is to separate $C_{0}(t)$ into single-solvent-molecule and solvent-pair contributions

$$
C_{0}(t)=C_{0}^{s}(t)+C_{0}^{p}(t)
$$

with the single-solvent-molecule term given by

$$
C_{0}^{s}(t)=\left\langle(\delta \Delta E)^{2}\right\rangle_{0}{ }^{-1} \sum_{j=1}^{N}\left\langle\delta \Delta w_{0 j}(0) \delta \Delta w_{0 j}(t)\right\rangle_{0}
$$

where $\delta$ denotes a fluctuation and $\Delta w_{0 j}$ is the change in the potential between the solute and the $j^{\text {th }}$ solvent molecule or ion. According to eq 8

$$
\Delta w_{0 j}=\sum_{\alpha \in 0} \sum_{\beta \in j} \frac{\Delta q_{0 \alpha} q_{j \beta}}{4 \pi \epsilon_{0}\left|\mathbf{r}_{0 \alpha}-\mathbf{r}_{j \beta}\right|}
$$

Figure 10 illustrates this decomposition of $C_{0}(t)$. In this case, as in previous studies of electrostatic $\mathrm{SD},{ }^{42,43} C_{0}^{s}(t)$ is positive and the pair term, $C_{0}^{p}(t)$ is negative. To display more clearly the relative magnitudes of the two terms and compare their time evolution, we plot $C_{0}^{s}(t)$ and $-C_{0}^{p}(t)$. The magnitudes of $C_{0}^{s}(t)$ and $-C_{0}^{p}(t)$ are very similar, especially at times $t>0.5 \mathrm{ps}$. In all cases, $C_{0}^{s}(t)$ and $-C_{0}^{p}(t)$ in the $0<t<2$ ps time interval are much larger than the resulting $C_{0}(t)$, which never exceeds $C_{0}(0)=1$, and are much more slowly decaying. In RMs, $C_{0}^{s}(t)$ and $-C_{0}^{p}(t)$ decrease with increasing water content. This is somewhat surprising, given that the number of molecular pairs increases with $w_{0}$, so one might have expected a larger value of $-C_{0}^{p}(t)$ if the number of solvent molecules within the RM were the only factor determining its size. The fact that the opposite trend is observed seems to indicate that the larger ionic concentration in the smaller RMs creates a more highly correlated solvent response to solute-induced electrostatic

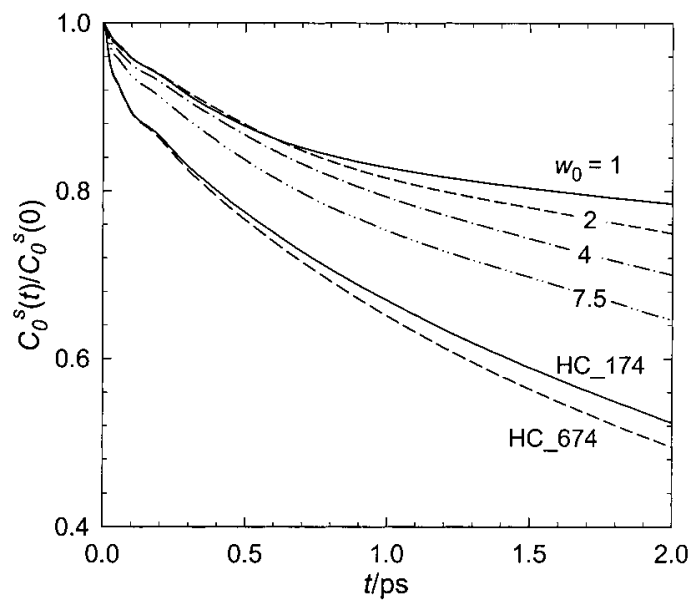

Figure 11. $C_{0}^{s}(t) / C_{0}^{\mathrm{s}}(0)$, the normalized single-solvent-molecule component of the ground-state solvation time correlation, $C_{0}(t)$, for the reverse micelles $w_{0}=1,2,4$, and 7.5 and for the two hydrophobic cavities HC_174 and HC_674.

perturbations. In the two $\mathrm{HCs}, C_{0}^{s}(t)$ and $-C_{0}^{p}(t)$ are nearly independent of cavity size, which further suggests that the solvation properties of these systems are near the large cavity limit. $C_{0}^{s}(t)$ and $-C_{0}^{p}(t)$ are still quite large, but appreciably smaller than in the $w_{0}=7.5 \mathrm{RM}$, confirming that the presence of ions leads to a more highly collective solvent response.

To clarify why the RM environment has less effect on SD than on the mobility of single molecules, we plot in Figure 11 $C_{0}^{s}(t) / C_{0}^{s}(0)$, the normalized single-molecule contribution to the solvation time correlation, $C_{0}(t) . C_{0}^{s}(t) / C_{0}^{s}(0)$ for the four RMs exhibits a much stronger dependence on $w_{0}$ than is found for the total solvation response, $S(t)$ or $C_{0}(t)$. Faster decay of $C_{0}^{S}(t) /$ $C_{0}^{s}(0)$ with increasing $w_{0}$ occurs on all time scales, even the inertial one for which the total solvent response shows essentially no $w_{0}$-dependence. In the two $\mathrm{HCs}$, the decay rate of $C_{0}^{s}(t) / C_{0}^{s}(0)$ is faster than in the largest $\mathrm{RM}, w_{0}=7.5$. Thus, reduced solvent mobility strongly affects the single-solventmolecule contribution to SD. It appears from Figure 11, however, that the highly collective nature of the RM solvation response, which becomes more collective as $w_{0}$ decreases, largely compensates for the reduced mobility of individual molecules, leading to a much weaker than expected effect on solvation properties. Further studies will be required to determine if this nearly complete cancellation is a specific feature of this model or a general property of SD in RMs and other complex interfacial systems.

\section{Summary and Conclusions}

We have presented here MD simulation results for solvation dynamics in model reverse micelles of varying size designed to represent water/AOT/oil microemulsions, in which the water pool is approximately spherical with a radius determined by $w_{0}$. These results were compared to solvation dynamics of water alone confined in spherical cavities of sizes resembling those of the larger RMs. Our simulations focused on the short-time dynamics of solvation, from 0 to $2 \mathrm{ps}$, a significant portion of which has not yet been accessed experimentally. We found that on this time scale the solvation response in RMs becomes faster as $w_{0}$ increases, in agreement with experiment, ${ }^{36,31-33}$ and as expected based on our previous simulation results on the $w_{0}$-dependence of water mobility. ${ }^{11}$ However, we find a $w_{0}$-dependence that is surprisingly weak, much weaker than what one might have expected on the basis of the strong 
$w_{0}$-dependence of the rotational and translational mobilities of water molecules in these model RMs. The $w_{0}$-dependence of $S(t)$ is especially weak over the $0-200 \mathrm{fs}$ time scale, over which $S(t)$ resembles the fast SD observed in bulk water. As in bulk water, in RMs and HCs much of the decay of $S(t)$, about $70 \%$ in our model systems, occurs on this time scale via inertial and librational mechanisms. We have carried out analyses of the nonequilibrium and the ground-state equilibrium MD trajectory data in order to explain these findings and have uncovered the following aspects of the solvation mechanism in RMs:

- The short-time solvation response is dominated by water. The response of the $\mathrm{Na}^{+}$counterions becomes important only on the subsequent diffusive time scale, which accounts for only about $20 \%$ of the decay of $S(t)$. The headgroups make a negligible contribution, given their low mobility. The relative importance of the $\mathrm{Na}^{+}$contribution to the steady-state Stokes shift decreases with increasing water content, $w_{0}$.

- For our model solute, much of the fast inertial response is due to the solute site that loses its partial charge in the $S_{1}$ state. All of the diffusive response is due to the solute site on which the negative charge localizes in $\mathrm{S}_{1}$ state. This is consistent with other simulations of SD in hydrogen-bonding solvents, which show that rapid inertial response follows a charge distribution change that breaks a solute-solvent hydrogen bond. ${ }^{66,67}$

- In the RMs, the solute is located in the core region. The fact that water in this region is more mobile than closer to the interface helps explain why $S(t)$ is less sensitive to the RM size than quantities that measure water mobility in the entire water pool.

- The linear response approximation to $S(t)$ and to the steadystate Stokes shift is quite accurate for RMs and somewhat less accurate, but still reasonable, for HCs. This approximation is expected to be even better for a model solute that resembles more closely the $\mathrm{C} 343$ chromophore, since the change in the solute-solvent interactions accompanying the $\mathrm{S}_{0} \rightarrow \mathrm{S}_{1}$ is more modest than for the present model solute.

- The solvent response is highly collective. This manifests itself as a high degree of cancellation of $C_{0}^{s}(t)$, the singlesolvent-molecule component of the solvation TCF by the negative solvent-pair component, $C_{0}^{p}(t)$. Even though the number of pairs of solvent molecules increases with increasing $w_{0}$, the extent of cancellation decreases. In the HCs, a smaller cancellation effect is found than even in the largest RM. This points to the important role of ions in making the solvent response more highly collective in RMs with higher ionic concentration. Decomposition of $C_{0}(t)$ into its major solvent components also points to the important role that the $\mathrm{Na}^{+}$ions play in the longer-time solvation dynamics, both through their autocorrelation and the negative $\mathrm{Na}^{+}-\mathrm{H}_{2} \mathrm{O}$ cross correlations.

Our finding that the short time dynamics of solvation are only weakly affected by the RM environment, though surprising, is in accord with experimental observations. The experiments to date have been unable to resolve the inertial portion of the solvent response, and have focused instead on the relatively small portion of the response that extends over a picosecond to nanosecond time scale. We also observe a long time tail in our response functions that indicates a slower water response in the RMs compared to both the HCs and bulk. This tail is largest, indicating slowest response, in the smallest RM and decreases as $w_{0}$ increases. The comparison between the RM and $\mathrm{HC}$ results further suggests that it is the highly ionic environment rather than confinement in a cavity that is primarily responsible for the slowdown of SD for this model chromophore. Although longer simulation runs will be required to determine how long these time scales extend, the current simulations already show roughly a factor of 4 difference in the amount of solvation response that occurs after 2 ps between the smallest RM and the HCs. There is thus a considerable slowing down in one portion of the solvation response in our model, even though we would characterize the full solvation response as being remarkably robust.

The simulations do not provide an explanation for the observation mentioned in the Introduction that the extent of the solvent response missed by the experiments increases as the RMs become smaller. ${ }^{40}$ In our model, the time scale for early SD remains fairly constant. We have observed, however, that the highly ionic environment increases collective nature of the response, and one explanation might be that this environment increases the coupling between the intramolecular and solvent modes, breaking the separation of time scales for the intramolecular and solvent relaxation, which is used to estimate the total solvent response. ${ }^{41}$

Two other groups have recently reported simulation results for SD in related systems. Senapati and Chandra ${ }^{45}$ have performed MD simulations of a Stockmayer liquid in smooth Lennard-Jones cavities. They report a significant slowing down of SD for the instantaneous charging of a neutral atom fixed near the cavity wall as compared with bulk. It appears most of the difference is due to a strong damping of the inertial response in the cavity, which may occur because the probe position is fixed. Although a direct comparison is not possible because the models are quite different, these results taken together with ours, where the probe molecule floats freely in the RM core, suggest that the location of the probe may strongly affect what dynamics are observed. The importance of probe location is reinforced by the findings of Balasubramanian and Bagchi, ${ }^{46}$ who modeled solvation dynamics at the surface of a normal micelle. This system provides a nice complement to ours because it is an example of water in a highly ionic environment without confinement. The used tagged water molecules and $\mathrm{Cs}^{+}$ counterions as SD probes, finding a modest slowdown in SD for the water probe and a very dramatic increase in solvation time ( $>100 \mathrm{ps}$ ) for $\mathrm{Cs}^{+}$. Decomposition of $C(t)$ (calculated from the fluctuations in the total probe-solvent electrostatic potential energy) however, revealed that nearly all of the slow dynamics for $\mathrm{Cs}^{+}$were due to interactions with other ions at the interface, and that the water component of the response was very small and relatively fast. These results, along with our finding that the $\mathrm{Na}^{+}$counterions made up a substantial and relatively slow portion of the RM response, suggest that interactions between the probe and the interface may comprise a significant portion of what is often labeled as "slow solvent response."

In the future, we plan to investigate in more detail the role of probe location. A stronger effect from the restricted water mobility might be observed in our model for chromophores that reside in the interfacial region because the concentration of ions is much higher and water mobility is more highly restricted. We also plan to try probes that more closely resemble the large aromatic dyes that are used in the experiments. It should also be noted that there are other dynamical processes in RMs that might contribute to SD at longer time scales, but have not been included in our model. The most important of these are likely to be shape deformations away from spherical symmetry, ${ }^{1,68}$ which influence the dielectric properties of the water pool. ${ }^{69,70}$ Developing a model which would take into account such structural deformations, without including an atomistic representation of the surfactant tails and the nonpolar solvent would be highly desirable. 
Acknowledgment. This work was supported in part by the National Science Foundation Grants CHE-9520619 and CHE9981539. We thank Professor Nancy E. Levinger for helpful discussions and for sharing with us her preliminary experimental results ${ }^{40}$ on the steady-state fluorescence Stokes shifts in the electronic spectra of C343 in water/AOT/oil reverse micelles. We are grateful to Professor Biman Bagchi and Dr. S. Balasubramanian for sending us a preprint of their manuscript on SD in micellar solutions. ${ }^{46}$ We also thank the two referees for careful reading of the manuscript and helpful comments. One referee in particular deserves credit for noticing that our original method of solute placement resulted in excessive removal of water molecules.

\section{References and Notes}

(1) de Gennes, P. G.; Taupin, C. J. Phys. Chem. 1982, 86, 2294.

(2) Langevin, D. Acc. Chem. Res. 1988, 21, 255.

(3) Chevalier, Y.; Zemb, T. Rep. Prog. Phys. 1990, 53, 279.

(4) Micelles, Membranes, Microemulsions, and Monolayers; Gelbart,

W. M., Ben-Shaul, A., Roux, D., Eds.; Springer: Berlin, 1994.

(5) Gelbart, W. M.; Ben-Shaul, A. J. Phys. Chem. 1996, 100, 13169.

99.

(6) Moulik, S. P.; Paul, B. K. Adv. Colloid Interface Sci. 1998, 78,

(7) Luisi, P. L.; Giomini, M.; Pileni, M. P.; Robinson, B. H. Biochim. Biophys. Acta 1988, 947, 209.

(8) Luisi, P. L.; Straub, B. E. Reverse Micelles: Biological and Technological Relevance of Amphiphilic Structures in Apolar Media; Plenum: New York, 1984.

(9) De, T.; Maitra, A. Adv. Colloid Interface Sci. 1995, 59, 95.

(10) Pileni, M. P. J. Phys. Chem. 1993, 97, 6961.

(11) Faeder, J.; Ladanyi, B. M. J. Phys. Chem. B 2000, 104, 1033.

(12) Eicke, H. F.; Rehak, J.; Phys.-Chem. Inst, U. B. B. S. Helv. Chim. Acta 1976, 59, 2883.

(13) Stratt, R. M.; Maroncelli, M. J. Phys. Chem. 1996, 100, 12981.

(14) Jimenez, R.; Fleming, G. R.; Kumar, P. V.; Maroncelli, M. Nature (London) 1994, 369, 471

(15) Lang, M. J.; Jordanides, X. J.; Song, X.; Fleming, G. R. J. Chem. Phys. 1999, 110, 5884.

(16) Bader, J. S.; Chandler, D. Chem. Phys. Lett. 1989, 157, 501.

(17) Maroncelli, M.; Fleming, G. R. J. Chem. Phys. 1988, 89, 5044.

(18) Levy, R. M.; Kitchen, D. B.; Blair, J. T.; Krogh-Jespersen, K. J. Phys. Chem. 1990, 94, 4470.

(19) Ando, K.; Kato, S. J. Chem. Phys. 1991, 95, 5966.

(20) Skaf, M. S.; Ladanyi, B. M. J. Phys. Chem. 1996, 100, 18258

(21) Re, M.; Laria, D. J. Phys. Chem. B 1997, 101, 10494.

(22) Tran, V.; Schwartz, B. J. J. Phys. Chem. B 1999, 103, 5570. 5382 .

(23) Aherne, D.; Tran, V.; Schwartz, B. J. J. Phys. Chem. B 2000, 104,

(24) Geissler, P. L.; Chandler, D. J. Chem. Phys. 2000, 113, 9759

(25) Bhattacharyya, K.; Bagchi, B. J. Phys. Chem. A 2000, 104, 10603.

(26) Zhang, J.; Bright, F. V. J. Phys. Chem. 1991, 95, 7900.

(27) Zhang, J.; Bright, F. V. J. Phys. Chem. 1992, 96, 9068.

(28) Zhang, J.; Bright, F. V. J. Phys. Chem. 1992, 96, 5633.

(29) Riter, R. E.; Undiks, E. P.; Levinger, N. E. J. Am. Chem. Soc. 1998, 120,6062 .

(30) Willard, D. M.; Riter, R. E.; Levinger, N. E. J. Am. Chem. Soc. 1998, 120, 4151.

(31) Riter, R. E.; Willard, D. M.; Levinger, N. E. J. Phys. Chem. B 1998, 102, 2705.
(32) Levinger, N. E.; Riter, R. E.; Willard, D. M.; Pant, D. Springer Ser. Chem. Phys. 1998, 63, 553.

(33) Pant, D.; Riter, R. E.; Levinger, N. E. J. Chem. Phys. 1998, 109, 9995.

(34) Riter, R. E.; Undiks, E. P.; Kimmel, J. R.; Levinger, N. E. J. Phys. Chem. B 1998, 102, 7931.

(35) Cho, C. H.; Chung, M.; Lee, J.; Nguyen, T.; Singh, S.; Vedamuthu, M.; Yao, S. H.; Zhu, J. B.; Robinson, G. W. J. Phys. Chem. 1995, 99, 7806.

(36) Sarkar, N.; Das, K.; Datta, A.; Das, S.; Bhattacharyya, K. J. Phys. Chem. 1996, 100, 10523

(37) Datta, A.; Mandal, D.; Pal, S. K.; Bhattacharyya, K. J. Phys. Chem. B 1997, 101, 10221 .

(38) Pal, S. K.; Mandal, D.; Bhattacharyya, K. J. Phys. Chem. B 1998, 102,11017

(39) Sarkar, N.; Datta, A.; Das, S.; Bhattacharyya, K. J. Phys. Chem. 1996, 100, 15483 .

(40) Levinger, N. E., Unpublished results.

(41) Fee, R. S.; Maroncelli, M. Chem. Phys. 1994, 183, 235.

(42) Ladanyi, B. M. In Electron Ion Transfer Condens. Media; Kornyshev, A. A., Tosi, M., Ulstrup, J., Eds.; World Scientific: Singapore, 1997; p 110 .

(43) Ladanyi, B. M.; Maroncelli, M. J. Chem. Phys. 1998, 109, 3204.

(44) Muiño, P. L.; Callis, P. R. J. Chem. Phys. 1994, 100, 4093.

(45) Senapati, S.; Chandra, A. J. Chem. Phys. 1999, 111, 1223.

(46) Balasubramanian, S.; Bagchi, B. 'Fast and slow dynamics of water near a micellar surface', preprint.

(47) Linse, P. J. Chem. Phys. 1989, 90, 4992.

(48) Linse, P.; Halle, B. Molecular Physics 1989, 67, 537.

(49) Lee, C. Y.; McCammon, J. A.; Rossky, P. J. J. Chem. Phys. 1984, $80,4448$.

(50) Berendsen, H. J. C.; Grigera, J. R.; Straatsma, T. P. J. Phys. Chem. 1987, 91, 6269.

(51) Schweighofer, K. J.; Essmann, U.; Berkowitz, M. J. Phys. Chem. B 1997, 101, 3793 .

(52) Song, X.; Chandler, D. J. Chem. Phys. 1998, 108, 2594.

(53) Benjamin, I.; Whitnell, R. M. Chem. Phys. Lett. 1993, 204, 45.

(54) Allen, M. P.; Tildesley, D. J. Computer Simulation of Liquids; Clarendon: Oxford, U.K., 1987.

(55) Berendsen, H. J. C.; Postma, J. P. M.; van Gunsteren, W. F.; DiNola, A.; Haak, J. R. J. Chem. Phys. 1984, 81, 3684.

(56) Bagchi, B. Annu. Rev. Phys. Chem. 1989, 40, 115.

(57) Barbara, P. F.; Jarzeba, W. Adv. Photochem. 1990, 15, 1.

(58) Simon, J. D. Pure Appl. Chem. 1990, 62, 2243.

(59) Rossky, P. J.; Simon, J. D. Nature (London) 1994, 370, 263.

(60) Maroncelli, M. J. Mol. Liq. 1993, 57, 1.

(61) Carter, E. A.; Hynes, J. T. J. Chem. Phys. 1991, 94, 5961.

(62) Hansen, J. P.; McDonald, I. R. Theory of Simple Liquids, Second ed.; Academic Press: London, 1986.

(63) Laria, D.; Skaf, M. S. J. Chem. Phys. 1999, 111, 300.

(64) Day, T. J. F.; Patey, G. N. J. Chem. Phys. 1999, 110, 10937.

(65) Neria, E.; Nitzan, A. J. Chem. Phys. 1994, 100, 3855.

(66) Phelps, D. K.; Weaver, M. J.; Ladanyi, B. M. Chem. Phys. 1993 176,575 .

(67) Fonseca, T.; Ladanyi, B. M. J. Mol. Liq. 1994, 60, 1.

(68) Brown, D.; Clarke, J. H. R. J. Phys. Chem. 1988, 92, 2881.

(69) Van der Linden, E.; Geiger, S.; Bedeaux, D. Physica A (Amsterdam) 1989, 156,130

(70) Mittleman, D. M.; Nuss, M. C.; Colvin, V. L. Chem. Phys. Lett. 1997, 275, 332 . 